\title{
Observations of real solitons
}

\section{Peter L. Christiansen}

Solitons are localized nonlinear waves that can propagate and interact like particles. Theoretical studies show that phenomena such as water waves, light pulses in optical fibres, magnetic-flux quanta in superconducting devices and coherent excitations of biomolecules can be solitons. Computer simulations show that solitons can form in the presence of such realistic features as frictional loss mechanisms, external driving forces and thermal fluctuations. The solitons will exist under these circumstances for sufficiently long to be important features in the time evolution of the wave excitations. Experimental demonstrations of soliton dynamics, however, are still scarce. Therefore, two recent papers by Fujimaki, Nakajima and Sawada ${ }^{1}$ and by Wu, Wheatley, Putterman and Rudnick ${ }^{2}$ showing solitons in real systems are most noteworthy.

The work by Fujimaki et al. deals with collision of solitons on an electronic Josephson transmission line (JTL), $1.8 \mathrm{~mm}$ long, composed of a sequence of 31 discrete Josephson junctions (interleaved superconducting and insulating layers). In the continuum version of the JTL, the Josephson effect (superconducting electrons tunnelling through the insulating layers) results from the weak coupling between pairs of superconducting thin films. This overlap geometry is modelled very accurately by the sine-Gordon equation originally developed by particle physicists. In 1962, Perring and Skyrme ${ }^{3}$ showed that this nonlinear partial differential equation possesses solutions that they termed 'kink' and 'antikink', after

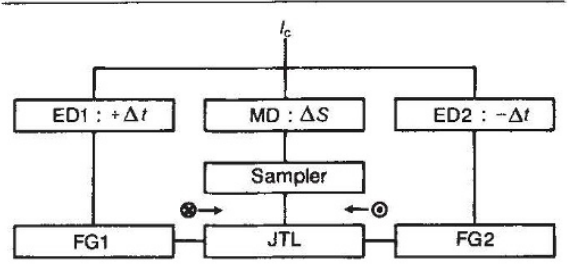

Fig. 1 Simplified block diagram of the fluxon experiment of Fujimaki et al. The fluxon and antifluxon (cross and dot, respectively) are injected into the Josephson transmission line (JTL, made of 31 Josephson junctions, each a $4 \times 4-\mu \mathrm{m}^{2}$ sandwich of two superconducting films and one insulating) by the fluxon generators FG1 and FG2. Electronic delays ED1 and ED2 delay the fluxon and advance the antifluxon by $\Delta t$, effectively shifting the system $v \Delta t$ leftwards along the JTL. The sampler records the flux density at the midpoint of the JTL after a variable mechanical time delay (MD) of $\Delta S$. The whole process is initiated when the control current $I_{\mathrm{c}}$ exceeds a critical value. (From ref.1.) their shapes, which can propagate and interact with each other completely nondestructively, suffering only phase shifts as a result of the interaction.

In certain respects, Perring and Skyrme's paper foreshadows the pioneering work by Zabusky and Kruskal on the Korteweg-de Vries equation (originally formulated to describe shallow water

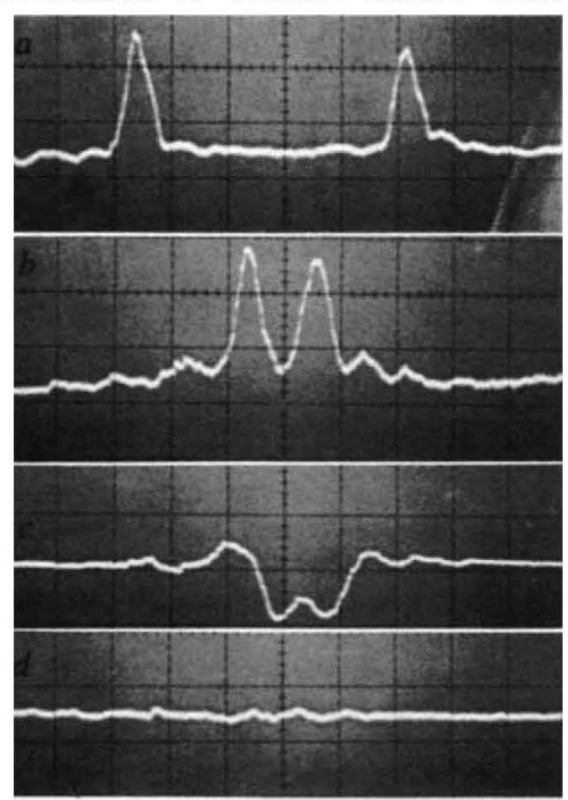

Fig. 2 Fluxon-antifluxon annihilation process observed $a 0, b 8, c 16$ and $d 24$ ps after the fluxons are launched. The width of the picture is $1.8 \mathrm{~mm}$. (Courtesy of A. Fujimaki.)

waves), which introduced the soliton concept. The derivative of Perring and Skyrme's kinks corresponds to the solitons. An isolated kink solution to the sine-Gordon equation cannot be destroyed and is therefore a 'topological' soliton. In the JTL theory, these solitons carry magnetic-flux quanta (current vortices) and are termed fluxons. Correspondingly, negative magnetic flux quanta are carried by antifluxons. One consequence of the theory is that the fluxons behave as relativistic particles (their effective mass increases with increased velocity). In both papers ${ }^{3,4}$ the analysis was directly inspired by computational results.

The superconducting circuit, made using standard lead-based technology, consists of two fluxon generators, the JTL and a sampler to measure the flux in the JTL (Fig. 1); all are based on the Josephson effect. A fluxon is injected into the JTL when a control current $I_{\mathrm{c}}$ to the fluxon generator exceeds a critical value. A bias current $I_{\mathrm{b}}$ perpendicular to the JTL controls the velocity $v$ of the fluxons (which is

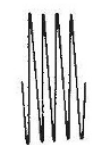

$240^{\circ}$

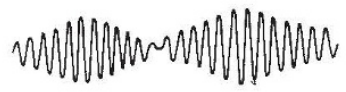

$185^{\circ}$

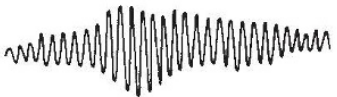

$120^{\circ}$

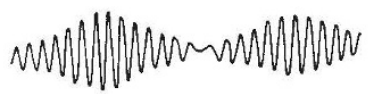

0

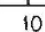

20

Time (ms)

Fig. 3 Collision of clockwise and anticlockwise envelope solitons travelling around a thin cylindrical shell. The shape of the envelope persists because of the balance between amplitude dispersion and the nonlinearity of the elastic medium. Without this balance the wave packet would disperse. The top trace shows the excitation pulse. (From ref. 2.)

typically $4 \times 10^{7} \mathrm{~m} \mathrm{~s}^{-1}$ ), and must also exceed a minimum value. The sampler detects only at one central point, so that the flux across the whole JTL cannot be measured in one go.

Instead, a variable delay $2 \Delta t$ can be introduced between the two fluxon generators, effectively moving the fluxonantifluxon pair left or right by a distance $v \Delta t$. A single scan, such as shown in Fig. 2, is obtained by sweeping $\Delta t$. The time sequence shown in Fig. 2 is obtained by introducing a second delay $\Delta S$ before firing the sampler.

Fujimaki et al. thus observe the collision of a fluxon and an antifluxon, in which the two merge, interact and dissipate their energy via a 'breather mode' (the soliton and antisoliton bound together in a localized, lower-energy state) into linear oscillations ('radiation') which are finally absorbed by shunt resistances (Fig. 2). This agrees well with computational results and can also be understood in terms of fluxon perturbation theorys. In other cases, the fluxons can survive the collision. The fringes in the traces are nonsolitonic effects inevitable in real versions of such idealized mathematical concepts. The authors also demonstrate the relativistic nature of the fluxons by showing that the product of the pulse height and the pulse width is independent of the fluxon velocity.

Wu et al. ${ }^{2}$ observed envelope solitons in elastic solids for the first time. In contrast to topological solitons, envelope solitons are wave packets with a solitonshaped envelope (Fig. 3). Earlier experiments with fluid surface waves have been 
interpreted in terms of envelope solitons ${ }^{6}$. Wu et al. ${ }^{2}$ excite flexural wave packets by an acoustic horn driver on a circularcylindrical thin elastic shell. The waves propagate clockwise and anticlockwise around the cylinder. Their nonlinear interaction is seen by means of transducers mounted on the shell. The reason for using thin shells is that this system is very dispersive and has a high nonlinear response, making the generation of solitons feasible, and a large quality factor, so that they persist. Such a combination of parameters generally makes soliton formation in off-equilibrium (highly distorted) systems accessible to observation.

As in other quickly varying hyperbolic classical fields, the slow modulations of the flexural waves on the shell are described by the nonlinear Schrödinger equation. The parameters in the interacting envelope soliton solutions of this equation can be fitted perfectly to the observed data, thus implying the existence of envelope solitons.

\section{Vaccine development}

\section{Antigenic hybrids of poliovirus}

\section{James M. Hogle}

At A recent meeting*, three research groups reported the successful construction of viable intertypic chimaeras (hybrids) of poliovirus. In each case, the infectious cloned complementary DNA encoding one of the three serotypes of the virus was modified so that the amino-acid sequence of one of the antigenic sites of one serotype is replaced with the analogous antigenic site from a different serotype. All three groups focused on a particular region of the virus capsid protein, VP1, that is known to be a dominant antigenic site of type 2 and type 3 poliovirus (see figure $)^{1}$. These studies have important implications for the design of novel vaccines for polio and related viruses, and on page 81 of this issue ${ }^{2}$, Burke et al. present the first published report of such an antigenic chimaera.

Poliovirus causes an estimated 250,000 $2,000,000$ cases of paralytic poliomyelitis annually, predominantly in unvaccinated or under-vaccinated children in developing countries. Introduction of both killed and live attenuated vaccines against poliovirus have resulted in the virtual elimination of poliomyelitis in industrialized countries. The live, attenuated polio vaccines are among the safest and most effective vaccines currently in use. Nonetheless, a handful of cases continue to occur each year in countries with extensive vaccination programmes. Virtually all

* Molecular aspects of picornavirus infection and detection. ICN-UCI international conference on virology, Newport Beach, California, 14-15 January 1988.
Solitons are not of interest to physicists alone. Davydov ${ }^{7}$ has proposed application of envelope solitons to understand energy Our computer studies ${ }^{8}$ of the quantum model of $\alpha$-helical proteins show that the solitons have long lifetimes at biological temperatures. Unfortunately, experimental evidence of this mechanism remains to be obtained.

Fujimaki, A., Nakajima, K. \& Sawada, Y. Phys. Rev. Lett. 59, 2895-2898 (1987).

Wu, J., Wheatley, J., Putterman, S. \& Rudnick, I. Phys. Rev. Lett 59, 2744-2747 (1987)

Perring, J.K. \& Skyrme, T.H.R. Nucl. Phys. 31, 550 (1962) 4. Zabusky, N.J. \& Kruskal, M.D. Phys. Rev. Lett. 15, 240 243 (1965).

. Pedersen, N.F., Samuelsen, M.R. \& Welner, D. Phys. Rev B30, 4057-4059 (1984)

6. Yuen, H.C. \& Lake, B.M. Phys. Fluids 18, 956-960 (1975) 7. Davydov, A.S. J. theor. Biol. 38, 559-569 (1973).

8. Cruzeiro, L., Halding, J., Christiansen, P.L., Skovgaard, O. \& Scott, A.C. Phys. Rev. A (in the press).

Peter L. Christiansen is at the Centre for Modelling Nonlinear Dynamics and Irreversible Thermodynamics (MIDIT), The Technical University of Denmark, DK-2800 Lyngby, Denmark. storage and transport in protein chains. these cases are associated with an increased neurovirulence of the types 2 and 3 attenuated Sabin vaccine strains on replication in vaccinees ${ }^{3}$. In contrast, there is no well-documented vaccineassociated case of poliomyelitis caused by the Sabin type 1 strain $^{3}$. Consistent with this observation, genetic studies of the vaccine strains show that only two of the genomic differences between the Sabin 3 strain and its neurovirulent parent Leon 3 contribute significantly to its attenuation ${ }^{4}$, whereas the Sabin strain of type 1 poliovirus has accumulated multiple attenuating mutations with respect to its neurovirulent parent (type 1 Mahoney) ${ }^{5}$. It has therefore been suggested that an appropriate grafting of neutralizing antigenic sites from the genetically less stable type 2 and type 3 strains into the stable attenuated background of type 1 poliovirus could provide a vaccine with a considerably lowered incidence of reversion to neurovirulence.

In their paper in this issue ${ }^{2}$, Burke et al. report the construction of an antigenic chimaera in which they replaced aminoacid residues $95-102$ of VP1 of the type 1 Sabin strain with the corresponding sequence from a variant of the type 3 Sabin strain. The chimaera was constructed by oligonucleotide-directed mutagenesis, and has the expected reactivities with panels of antisera and with panels of monoclonal antibodies against type 1 and type 3 poliovirus. The chimaera produced titres (measured in an antigenblocking assay) against both type 1 and type 3 poliovirus in rabbits and mice, and against both type 1 and type 3 poliovirus after oral administration to a cynomolgus monkey.

Murray, Kuhn and Wimmer (State University of New York at Stony Brook), collaborating with Kawamura and Nomoto (Tokyo Metropolitan Institute of Medical Science) and with Arita (Japanese

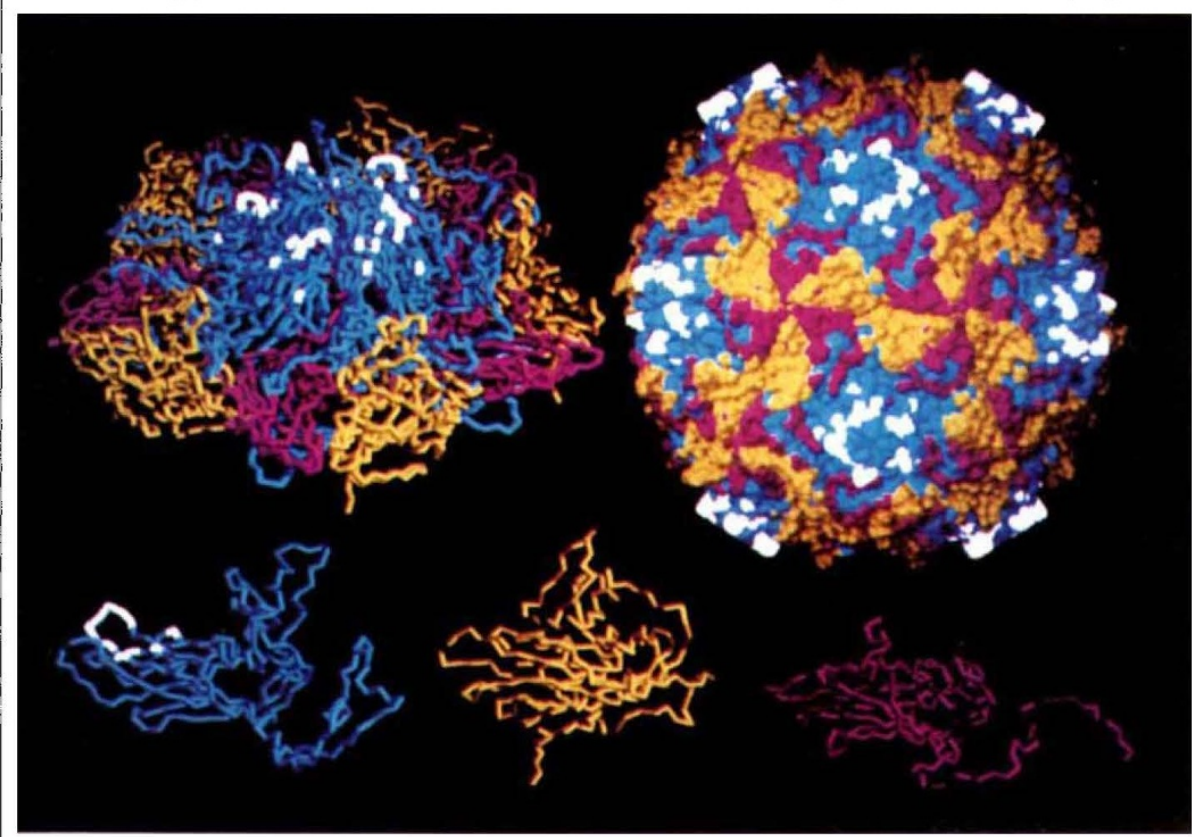

The region that has been replaced in the antigenic chimaeras described here is a 'loop' in the threedimensional structure of the Mahoney strain of type 1 poliovirus ${ }^{8}$. Capsid proteins VP1, VP2 and VP3 are blue, yellow and red, respectively. The substituted loop in VP1 is highlighted in white. The internal protein VP4 is not shown. Alpha-carbon models of the individual capsid proteins are shown at the bottom; a pentamer is shown at the top left; an intact virion is shown at the top right. Note that five copies of the substituted loop cluster near each of the 5-fold axes of the particle. 